\title{
Review of existing experimental methods for assessing the outcome of plant food supplementation on immune function
}

\author{
Dalu Mancama ${ }^{a, *}$, Dashnie Naidoo $^{a}$, Patrizia Restani ${ }^{b, 1}$, Paolo Meoni ${ }^{c, 2}$

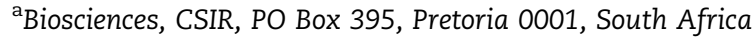 \\ bipartemente Scienze Farmacologiche e Biomolecolari, Università degli Studi di Milano, Italy \\ ${ }^{c}$ Atunis Development Services, Centre Phenicia, 2070 La Marsa, Tunisia
}

\section{A R T I C L E I N F O}

\section{Article history:}

Received 4 April 2013

Received in revised form

4 July 2013

Accepted 8 July 2013

Available online 1 August 2013

Keywords:

Plant food supplement

Immune function

Biomarkers

Analytical methods

Benefit

Clinical ratings scale

\begin{abstract}
A B S T R A C T
Plant food supplements (PFS) have become increasingly popular with respect to their consumption for improving human immune function. Despite this popularity, critical review is lacking regarding the analytical methods used to assess PFS outcome. The suitability of such methods for clinical-based studies remains particularly unclear. We undertook a literature-based review of the methods used to assess PFS outcome in immune function, to identify and assess the relevance of different technologies. Most methods described in this review adequately measured the functions of innate and adaptive immunity, were applicable to both healthy and diseased subjects, and were appropriate for assessing the benefit claims of PFS on immune function. However, the design and reporting quality of studies varied widely across trials, in some cases potentially impacting negatively on the outcomes and interpretations. Several strategies to enhance study robustness and quality were outlined, to improve the validity of the data generated in the field.
\end{abstract}

(c) 2013 Elsevier Ltd. All rights reserved.

\section{Contents}

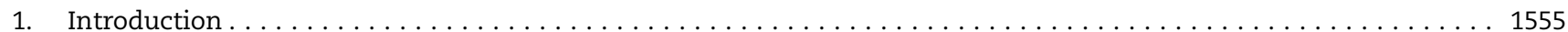

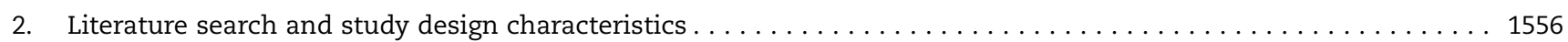

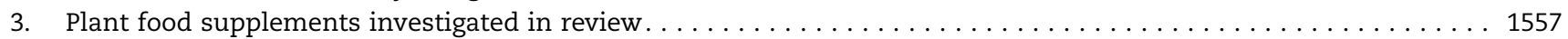

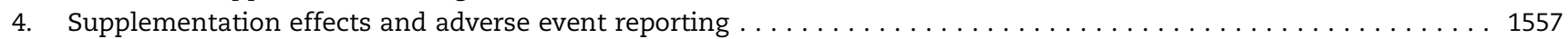

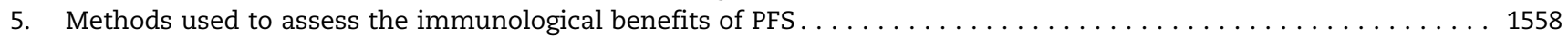

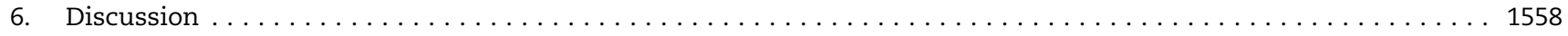

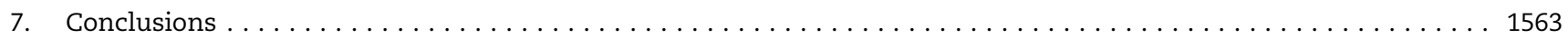

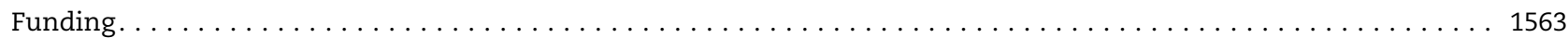

\footnotetext{
* Corresponding author. Tel.: +27 12 8414533; fax: +27 128414790.

E-mail addresses: dmancama@csir.co.za (D. Mancama), dnaidoo2@csir.co.za (D. Naidoo), patrizia.restani@unimi.it (P. Restani), meonip@atunis-development.com (P. Meoni).

1 Tel.: +39 0250318371; fax: +390250318284

2 Tel.: +216 71728796 .

1756-4646/\$ - see front matter (c) 2013 Elsevier Ltd. All rights reserved.

http://dx.doi.org/10.1016/j.jff.2013.07.008
} 


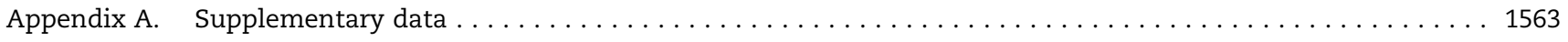

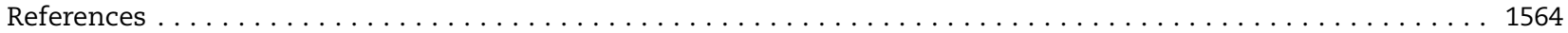

\section{Introduction}

The human immune system is comprised of networks of cells, tissues, and organs, which together represent the body's primary mechanism for preventing diseases that arise through toxins or infection from pathogenic microorganisms' including bacteria, viruses and fungi (Parham, 2009). To prevent or overcome infection the immune system is therefore required to function fast and effective against a range of pathogens, often under varying environmental (e.g. seasonal changes) or physiological (e.g. stress) conditions. The functions of the immune system are categorised into two major components, innate response and adaptive response, each of which is classified according to the speed and specificity of the immune reaction. Innate immunity encompasses cellular elements such as neutrophils, monocytes, macrophages, cytokines, and serum complement proteins, which provide immediate sensing and host defence against pathogens (Bonilla \& Oettgen, 2010). These elements rely on recognising the presence of pathogens or their products through the initial binding of soluble proteins and cell-surface receptors which then facilitate the process of elimination (Bonilla \& Oettgen, 2010; Parham, 2009). At the same time humoral elements also contribute significantly, in particular antimicrobial peptides such as defensins, glycoproteins and proteoglycans. In addition, the body is host to symbiotic communities of benign microbial flora that prevent infection by colonising tracts, and producing antimicrobial proteins such as colicins that incapacitate other bacteria (Di Meglio, Perera, \& Nestle, 2011; Parham, 2009; Turvey \& Broide, 2010).

In contrast, adaptive immunity results from antigenspecific reactions facilitated through $\mathrm{T}$ and $\mathrm{B}$ lymphocytes in two main stages, and is characterised by a slow precise adaptive response that develops over a period of days to several weeks. In the initial stage pathogen-specific cell surface receptors are used to recognise the invading microorganisms, triggering the proliferation and differentiation of large copies of pathogen-specific effector cells as part of the primary immune response. Following pathogen elimination a proportion of lymphocytes persist in the body, retaining immunological memory that can be elicited through a faster secondary immune response to eliminate the same pathogen in the event of subsequent infections (Bonilla \& Oettgen, 2010; Parham, 2009). Occasionally, in-borne errors in immune function occur, leading to the development of immune disorders including asthma, Crohn's disease (inflammatory bowel disease), inflammatory arthritis, and autoimmune diseases such as polyglandular syndrome and aspects of diabetes that result from the immune system attacking host cells (Dessein, Chamaillard, \& Danese, 2008; Finn \& Bigby, 2009; Kim, DeKruyff, \& Umetsu, 2010; Mizuno, 2006; Obermayer-Straub, Strassburg, \& Manns, 2000; Sly \& Holt, 2011; Van Heel, McGovern, \& Jewell, 2001). In rare instances severe combined immunodeficiency (SCID) can occur, which represents a group of sometimes fatal congenital disorders characterised by little or no immune response (Pearson, Greiner, \& Shultz, 2008).

Multiple factors such as genetic predisposition, dietary intake, and stress, influence human immune function (Gleeson, 2005; Hughes, 1999; Ibs \& Rink, 2003; Lomax \& Calder, 2009; O'Leary, 1990; Plat \& Mensink, 2005; Webster Marketon \& Glaser, 2008). To preserve optimal immune function, plant food supplements (PFS) have in recent years been increasingly consumed (Canter \& Ernst, 2004; Cassileth, Heitzer, \& Wesa, 2009). While terms such as PFS, Botanicals, Nutraceuticals, Functional Foods, Pharmafoods and others are sometimes used interchangeably, the Directive 2002/46/EC of the European Parliament and of the Council defines food supplements (FS) as "the foodstuffs for which the purpose is to supplement the normal diet and that are concentrated sources of nutrients or other substances with a nutritional or physiological effect, alone or in combination, marketed in dose form, namely forms such as capsules, pastilles, tablets, pills and other similar forms, sachets of powder, ampoules of liquids, drop dispensing bottles, and other similar forms of liquids and powders designed to be taken in measured small unit quantities. Plant Food Supplements (PFS) is a type of food supplement (FS) in which botanicals preparations are the main ingredients" (European Commission., 2002). From a regulatory perspective PFS are assimilated to foodstuff, and the benefit health claims allowed in the commercialisation of these products should exclude medical claims such as the prevention, diagnosis and treatment of a recognised pathological state. It is clear however that from the view-point of consumers, improved regulatory and procedural frameworks regarding health claims in general are still required given existing knowledge, perceptions, attitudes, beliefs and behaviour (Pothoulaki \& Chryssochoidis, 2009). This situation clearly requires very careful definition of the population assessed in clinical trials of PFS as well as the methods to be used in the clinical evaluation of the benefits related to the use of PFS, thus motivating this review. While a range of methodologies exist to evaluate the claimed benefits of PFS on immune function, critical review of the underlying supporting data and the relevance and utility of each method remains sparse. A major challenge in assessing PFS' benefits in the area of the immune system is also posed by the vast complexity of this system.

In this review we examined data contained in peerreviewed publications of clinical trials involving human subjects and assessed, primarily, the methodologies used to characterise the beneficial claims and effects of PFS on the human body. A concise summary of this work is illustrated in Fig. 1. Data for the review was sourced from the NCBI PubMed database and included studies published between January 1990 and July 2011. In order to provide the broadest possible unbiased overview of this area, data generated by non-randomised and / or controlled trials was also considered in order to allow for the limitations of this data to be discussed. Biochemical methods which assess immune function-related 


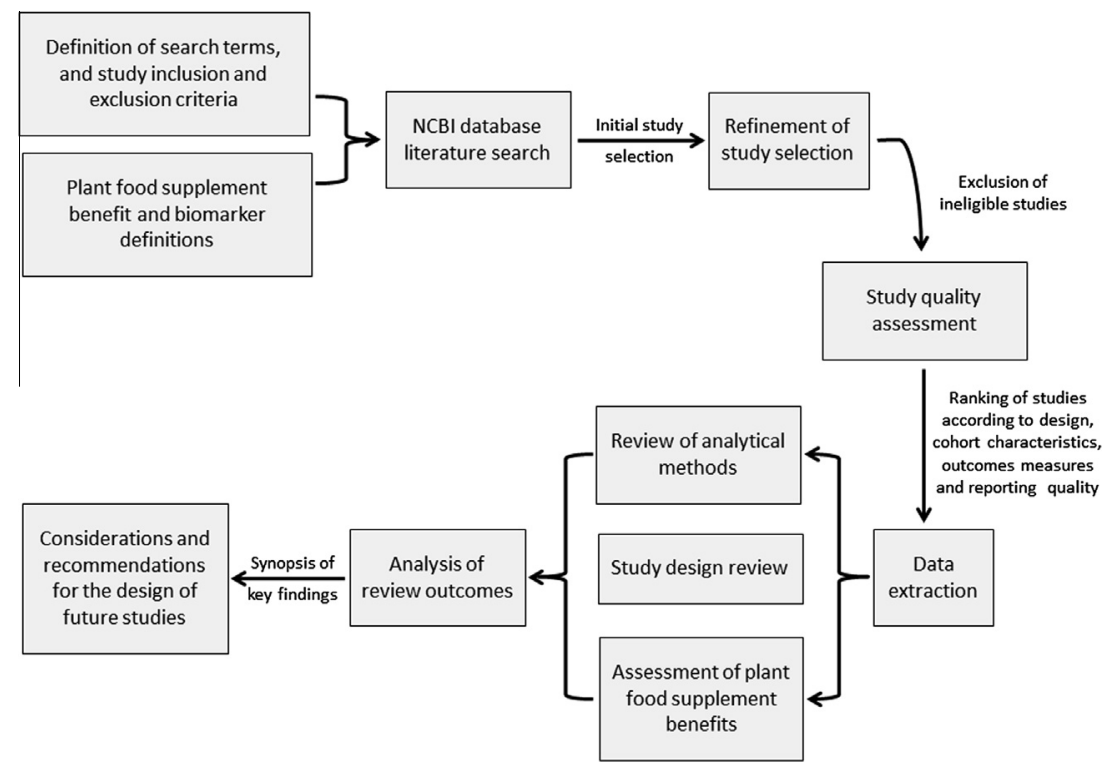

Fig. 1 - Schematic summary of the research strategy and key outcomes described in this review.

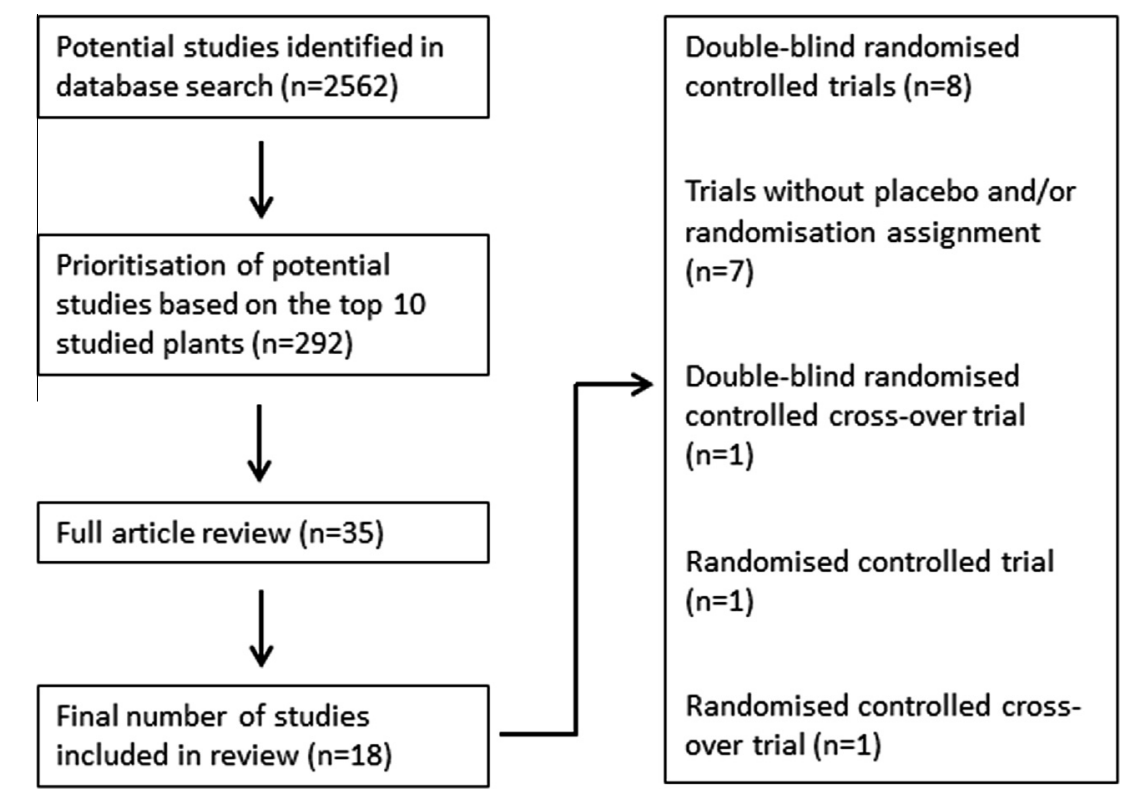

Fig. 2 - Illustration of the literature search performed to identify suitable studies for inclusion.

biomarkers, and clinical tools and methods were both considered in the review. Based on our findings recommendations for future research strategies are proposed, together with guidelines that may assist in harmonising methodologies used to determine the effects and benefit claims of plant food supplements on immune function.

\section{Literature search and study design characteristics}

The literature search interrogated immune function terms included in the article title, abstract, keywords and MeSH terms crossed with plant names and synonyms from the EuroFIR database, resulting in the identification of 2562 studies of potential relevance to this review (Kiely et al., 2010). The results of the literature search are summarised in Fig. 2. Based on the number of studies identified per plant species, the 10 plants most extensively studied for their effects on the immune system were prioritised as reported below. In total these plants were represented in 292 (11\%) of the 2562 potentially relevant studies. In order to be selected for further analysis, studies had to (i) primarily concern the clinical evaluation of PFS, (ii) include the evaluation of beneficial effects of PFS on biomarkers of the immune system, (iii) be published in the English Language, (iv) be representative of the most commonly studied plant ingredients within PFS. All studies that met these inclusion criteria and related to the most extensively studied plants were further screened by title 
and abstract for eligibility, resulting in 35 studies; the full article of each study was subsequently retrieved for detailed examination prior to selecting the final subset of studies for extraction. Full article reviews were not possible for 7 articles published in a language other than English, 4 articles were not available from source, while a further 6 did not meet the criteria for PFS. A total of 18 articles were eventually included in the final data extraction set. In order of prevalence 8 of these were based on double-blind randomised controlled trials (Alvarez-Cuesta et al., 2005; Bergendiova, Tibenska, \& Majtan, 2011; Bobovcak, Kuniakova, Gabriz, \& Majtan, 2010; Briviba et al., 2004; Rowe, Nantz, Bukowski, \& Percival, 2007; Savolainen, Jacobsen, \& Valovirta, 2006; Savolainen et al., 2007; Tang et al., 2009); 7 trials were conducted without placebo and/or randomisation assignment (Barak, Birkenfeld, Halperin, \& Kalickman, 2002; Beltran-Debon et al., 2010; Calabrese et al., 2000; Markovits, Ben Amotz, \& Levy, 2009; Myers et al., 2010; See, Mason, \& Roshan, 2002; Watzl, Bub, Brandstetter, \& Rechkemmer, 1999); and the remainder were based on studies of the following designs: a double-blind randomised controlled cross-over trial (Riso et al., 2006), a randomised controlled trial (Karlsen et al., 2010), and a randomised controlled cross-over trial (Wood, Garg, Powell, \& Gibson, 2008). Though the study inclusion criteria was as broad as possible and criteria did not exclude specific trial designs, not all trial designs were encountered in the final 18 selected studies. The overall quality of study design, as assessed by a methodological quality score was found to vary widely, with 3 being the highest and 0 the lowest score recorded. Not surprisingly, the lowest scoring studies generally reflected those without placebo and/or randomisation assignment. Overall the majority of studies were assessed to be of moderate to high quality (see Supplementary Table 1). Most studies incorporated the use of a placebo as the baseline comparator for establishing efficacy while 5 employed baseline values. In a single study a well-established reference supplement was employed against which test PFS were compared (see Supplementary Table 1). In total 523 participants were enroled across the 18 studies with the smallest study containing 8 participants and the largest containing 62 (Karlsen et al., 2010; Markovits et al., 2009).

\section{Plant food supplements investigated in review}

The potential studies identified in database searches were screened and ranked based on the plant active ingredient, to identify the plants with the highest number of published reports. The 10 most commonly studied PFS claimed to improve or enhance immune function identified in this study were: Andrographis paniculata (Green Chirayta) (Calabrese et al., 2000; See et al., 2002), Camellia sinensis (Tea) (Myers et al., 2010; Rowe et al., 2007), Corylus avellana (Common Hazel) (Savolainen et al., 2006; Savolainen et al., 2007), Garcinia mangostana (Purple Mangosteen) (Tang et al., 2009), Hibiscus sabdariffa (Roselle) (Beltran-Debon et al., 2010), Lycopersicon esculentum (Tomato) (Briviba et al., 2004; Markovits et al., 2009; Riso et al., 2006; Watzl et al., 1999; Wood et al., 2008), Olea europaea (Olive) (Alvarez-Cuesta et al., 2005), Pleurotus ostreatus (Oyster Mushroom) (Bergendiova et al., 2011; Bobovcak et al., 2010), Sambucus nigra (Elderberry) (Barak et al., 2002), and Vaccinium myrtillus (Bilberry) (Karlsen et al., 2010). The widespread consumption of several PFS obtained from these plants for enhancing immune function is well documented (Cassileth et al., 2009). PFS in the reviewed studies were administered either orally in capsule form, sublingually, or parenterally through injections. In the majority of trials (twelve in total), PFS formulations were sourced from commercial manufacturers and suppliers; in six trials the manufacturing source of the formulation was not stipulated. The majority of trials investigated single formulation supplements in capsule, liquid, or injectable form, most of which were sourced from commercial suppliers. In a minority of studies supplement formulations included additional PFS or minerals as part of the treatment regime. None of the additional constituents were reported to have a significant effect on the observed clinical outcomes, however in a number of studies such effects were not directly investigated and this potential could not be assessed or ruled out.

\section{Supplementation effects and adverse event reporting}

The inclusion criteria for the study population and the clinical outcomes being assessed varied widely across studies (Supplementary Table 1); these included common cold and flu, severe viral infection, immunomodulated symptoms that included allergy, and systemic inflammation. More appropriately considering the definition of PFS, the majority of studies (eleven out of the eighteen studies identified) investigated the direct effects of PFS in non-symptomatic cohorts, using genetic, protein and cellular biomarkers commonly associated with immune function as the underlying end-point indicator. For this review the definition of biomarker was defined according to the Biomarkers Definitions Working Group, whereby a biomarker is "a characteristic that is objectively measured and evaluated as an indicator of normal biological processes, pathogenic processes, or pharmacological responses to a therapeutic intervention" (Biomarkers Definitions Working Group. 2001). The key effects observed on immune function following PFS are summarised in Supplementary Table 2. In order to limit these observations to studies that inherently provide a higher level of confidence, studies in the table represent those based solely on randomised controlled trials. Whilst significant effects were observed in all of these studies the scope and magnitude of these effects varied considerably. For example supplementation with G. mangostana resulted in a small but statistically significant increase over baseline in double positive (DP) T cell frequency $(0.28 \%)$ compared to a decrease of $0.18 \%$ in the control group $(p=0.038)$. In contrast a relatively large effect was observed for L. esculentum, the use of which resulted in a significant decrease $(34.4 \%, p<0.05)$ compared to placebo in tumour necrosis factor (TNF) alpha production, which is an important immune cytokine involved in systemic inflammation (Tang et al., 2009). Supplementation with this PFS was also observed to induce significant decreases $(20 \%, p=0.009)$ in IL-4 production levels, which is a key regulator involved in adaptive 
immune function. In most studies non-significant effects of PFS on other specific immune functions were also observed.

Overall, the majority of studies did not report on the nature or prevalence of adverse events observed during or after PFS exposure. In instances where these events were reported symptoms included allergic reaction, fatigue, headache, pluritis or rash, loose stools or diarrhoea, nausea, metallic taste, bitter taste, decreased or no taste, dry tongue, decreased sex drive, eyes sensitive to light, decreased short term memory, dizziness, heartburn, tender lymph nodes, and lymphadenopathy in the case of A. paniculata supplementation; localised skin reactions following 0 . europaea immunotherapy; and diarrhoea, mild skin rash, gastric upset, dizziness, skin rash, and constipation in the case of $C$. sinensis supplementation. Interestingly a number of $C$. sinensis-related symptoms were also noted in the placebo group (Alvarez-Cuesta et al., 2005; Calabrese et al., 2000; Myers et al., 2010; Rowe et al., 2007). No adverse events were observed amongst participants receiving PFS derived from G. mangostana, L. esculentum, or P. ostreatus (Bergendiova et al., 2011; Briviba et al., 2004; Tang et al., 2009). Treatment duration was observed to vary widely, from regimes lasting a month or shorter (Beltran-Debon et al., 2010; Briviba et al., 2004; Karlsen et al., 2010; Markovits et al., 2009; Riso et al., 2006; Tang et al., 2009; Wood et al., 2008) to longer-term regimes lasting up to two years (Savolainen et al., 2006; Savolainen et al., 2007).

\section{Methods used to assess the immunological benefits of PFS}

A wide range of methods were identified that are used to assess the potential benefits of PFS on immune function, and these could be clearly categorised into bioanalytical approaches and non-invasive clinical tools and rating scales (summarised in Table 1). In the case of well-designed trials based solely on a randomised, blinded, controlled design, the selected methods could confirm at statistically significant levels, the claimed PFS benefit (s) on specific outcomes of immune function. It is interesting and relevant to note that in the cases where such claims could not be supported by the methods used, the respective groups had chosen to employ an open study design (Barak et al., 2002; Markovits et al., 2009). While the number of studies included in this review is limited in size, this observation raises the question about the extent to which failure to observe a therapeutic effect in open studies may be due to limitations in study design rather than a true lack of PFS efficacy. Clearer certainty could potentially be achieved by choosing to employ a study design based on a more robust randomised, blinded, controlled approach. The most cited bioanalytical method was based on immunoassay technology, with the immunoassay enzyme-linked immunosorbent assay (ELISA) representing the most popular approach (Engvall \& Perlmann, 1971; Riso et al., 2006; Rowe et al., 2007; See et al., 2002; Watzl et al., 1999; Wood et al., 2008). In the studies reviewed, this method was often employed for measuring circulating levels of a broad range of cytokines (e.g. TNF-alpha and multiple interleukins) and soluble cytokine receptors (e.g. TNF receptor type-1) (Table 1).
Another frequently cited method was flow cytometry, which was mainly employed to quantitatively measure changes to immune function-related cell numbers following PFS supplementation. This typically involved determining absolute counts of cells associated with innate response such as neutrophils, monocytes, macrophages and cytokines, and adaptive immune response processes e.g. T and B lymphoctyes (Bergendiova et al., 2011; Bobovcak et al., 2010; Calabrese et al., 2000; Myers et al., 2010; Rowe et al., 2007; Tang et al., 2009). In a number of studies the percentage contribution or ratio of each subclass to the total cell count was also used as the primary measure (Table 1) (Rowe et al., 2007). The method has also been adapted to measure cytokines (e.g. TNF-alpha and interleukins) and soluble cytokine receptors (e.g. TNF receptor type-1), as well as measuring the phagocytic activity of granulocytes and monocytes (Table 1). Variations to conventional flow cytometry included the upstream integration of solid phase immunoassay-based protein arrays which employ spectrally encoded antibody-conjugated beads designed for use in single or multiplexed assays (Beltran-Debon et al., 2010; Karlsen et al., 2007; Karlsen et al., 2010). The assay is performed in 96-well plate format and is read using flow cytometry-based technology that provides levels of quantitation and sensitivity comparable to ELISA. Biomarkers typically investigated through this approach include interleukins (e.g. Il-6 and Il-8) and the monocyte chemoattractant protein-1 (MCP-1).

The quantitative Real-Time polymerase chain reaction method (qRT-PCR) was another commonly employed technique, which is used to amplify and facilitate the detection of constitutive mRNA and/or DNA species representing immune biomarkers or pathogenic organisms of interest (Calabrese et al., 2000; Mullis et al., 1986; Savolainen et al., 2006; Savolainen et al., 2007). The method was found to be effective for quantifying relative interleukin levels (e.g. Il-4, Il-5, and Il-10), and levels of interferon (e.g. IFN-gamma) and related growth factors (e.g. TGF-beta) (Savolainen et al., 2006; Savolainen et al., 2007). The method was also capable of reliably quantifying HIV-1 copy numbers (Calabrese et al., 2000). Equally common were microscopy-based methods which are used to visually quantitate immune biomarkers (for example lymphocyte levels), typically through the use of haemocytometer counts (Bobovcak et al., 2010; Tang et al., 2009). The least cited method was nephelometry, which exploits the properties of light-scatter emitted by turbid samples to analyse macromolecules, for example total serum immunoglobulins (DeGrella et al., 1985; Tang et al., 2009). Non-invasive methods included direct skin reaction tests measured by planimetry using computerised scanners, and the use of clinical rating scales for quality of life measurements, for example non-hayfever symptoms, nasal symptoms, eye symptoms, and overall quality of life following the intake of PFS (Alvarez-Cuesta et al., 2005; Savolainen et al., 2006; Savolainen et al., 2007).

\section{Discussion}

The focus of this review was to assess the current methodologies used to characterise the benefit claims of PFS on human 
Table 1 - Current methods used to assess the potential benefits of plant food supplements on immune function. Key biomarkers and clinical outcomes are listed

according to the method used. Common advantages and disadvantages associated with the use of each method are also described.

\begin{tabular}{|c|c|c|c|c|}
\hline Method & References & $\begin{array}{l}\text { Immune biomarkers/clinical } \\
\text { variables measured }\end{array}$ & Advantages & Disadvantages \\
\hline $\begin{array}{l}\text { Immunoassay } \\
\text { (Direct ELISA) }\end{array}$ & See et al. $(2002)^{*}$ & $\begin{array}{l}\text { TNF-alpha, and soluble TNF receptor } \\
\text { type-I }\end{array}$ & $\begin{array}{l}\text { Fast, minimal preparation and can be } \\
\text { performed on whole blood, medium } \\
\text { sample throughput, reasonably low } \\
\text { sample volume requirements, no } \\
\text { secondary antibody cross reactivity }\end{array}$ & $\begin{array}{l}\text { Enzyme labelling may affect primary } \\
\text { antibody immunoreactivity; minimal } \\
\text { signal amplification; relatively } \\
\text { expensive approach which requires } \\
\text { primary antibody labelling for each } \\
\text { specific ELISA assay }\end{array}$ \\
\hline (Sandwich ELISA) & Rowe et al. (2007) & $\begin{array}{l}\text { IFN-gamma levels from PBMC culture } \\
\text { supernatants }\end{array}$ & $\begin{array}{l}\text { Fast, can be performed on whole blood, } \\
\text { medium sample throughput, reasonably } \\
\text { low protein and sample volume } \\
\text { requirements, good sensitivity and } \\
\text { selectivity, circumvents creation of } \\
\text { enzyme-linked antibodies for every } \\
\text { antigen of interest }\end{array}$ & $\begin{array}{l}\text { Potential for cross-reactivity with } \\
\text { secondary antibody, longer incubation } \\
\text { and processing step required }\end{array}$ \\
\hline (Sandwich ELISA) & Tang et al. (2009) & $\begin{array}{l}\text { Serum interleukins (IL-1alpha, IL-1beta, } \\
\text { and IL-2) }\end{array}$ & $\begin{array}{l}\text { Fast, can be performed on whole blood, } \\
\text { medium sample throughput, reasonably } \\
\text { low protein and sample volume } \\
\text { requirements, good sensitivity and } \\
\text { selectivity, circumvents creation of } \\
\text { enzyme-linked antibodies for every } \\
\text { antigen of interest }\end{array}$ & $\begin{array}{l}\text { Potential for cross-reactivity with } \\
\text { secondary antibody, longer incubation } \\
\text { and processing step required }\end{array}$ \\
\hline (Direct ELISA) & Wood et al. (2008) & Neutrophil elastase & $\begin{array}{l}\text { Fast, minimal preparation and can be } \\
\text { performed on whole blood, medium } \\
\text { sample throughput, reasonably low } \\
\text { sample volume requirements, no } \\
\text { secondary antibody cross reactivity }\end{array}$ & $\begin{array}{l}\text { Enzyme labelling may affect primary } \\
\text { antibody immunoreactivity; minimal } \\
\text { signal amplification; relatively } \\
\text { expensive approach which requires } \\
\text { primary antibody labelling for each } \\
\text { specific ELISA assay }\end{array}$ \\
\hline (Direct ELISA) & Watzl et al. (1999) & Lymphocyte and IL-2/IL-4 production & $\begin{array}{l}\text { Fast, minimal preparation and can be } \\
\text { performed on whole blood, medium } \\
\text { sample throughput, reasonably low } \\
\text { sample volume requirements, no } \\
\text { secondary antibody cross reactivity, } \\
\text { measures cell proliferation by } \\
\text { quantitating 5-bromo-2-deoxyuridine } \\
\text { incorporated into newly synthesized cell } \\
\text { DNA; good sensitivity }\end{array}$ & - \\
\hline (Sandwich ELISA) & Barak et al. (2002) & $\begin{array}{l}\text { Cytokine production (IL-1b, TNFa, IL-6, IL- } \\
8 \text {, IL-10) }\end{array}$ & $\begin{array}{l}\text { Fast, can be performed on whole blood, } \\
\text { medium sample throughput, reasonably } \\
\text { low protein and sample volume } \\
\text { requirements, good sensitivity and } \\
\text { selectivity, circumvents creation of } \\
\text { enzyme-linked antibodies for every } \\
\text { antigen of interest }\end{array}$ & $\begin{array}{l}\text { Potential for cross-reactivity with } \\
\text { secondary antibody, longer incubation } \\
\text { and processing step required }\end{array}$ \\
\hline
\end{tabular}


(Sandwich ELISA)

Karlsen et al. (2010)

Flow cytometry

(Bead-array flow cytometry)

Beltran-Debon et al. (2010)

Bobovcak et al. (2010)

Bergendiova et al. (2011)

Riso et al. (2006)

Tang et al. (2009)

Myers et al. (2010)
IL-1beta, IL-1alpha, IL-1 receptor antagonist (IL-1Ra), IL-2, IL-2R IL-4, IL-5, IL-6, IL-7, IL-8, IL-10, IL-12, IL-13, IL-15, IL17, TNF-alpha, interferon (IFN)-alpha, IFN-gamma, granolyte/macrophage colony-stimulating factor (GM-CSF), macrophage inflammatory protein 1alpha and 1-beta, immunoprotein (IP)-10 monocyte chemoattractant protein(MCP-1), monokine induced by IFNgamma (MIG), eotaxin and regulated upon activation

Normal T cell expressed and secreted (RANTES) measured in heparin plasma

Granulocyte and monocyte phagocytic activity

Ratio of alpha-beta and gamma-delta $\mathrm{T}$ cells in PBMC suspensions

Gamma-delta-T cells (CD3+, CD4-, CD8-), T-helper cells (CD3+, CD4+, CD8-), cytotoxic T cells (CD3+, CD4-, CD8+), CD4/CD8 double-positive T cells (CD3+, CD4+, CD8+), and Th to Tc cell ratio (Th/ Tc).

IL-6, IL-8, and MCP-1 measured in supernatants and plasma aliquots

Leucocyte and lymphocyte counts; NKcell activity

Phagocytosis and NK cell count

IFN-gamma and TNF-alpha production

Lymphocyte counts

Total white blood cell and lymphocyte numbers as a percentage of total blood cells
Fast, can be performed on whole blood, medium sample throughput, reasonably low protein and sample volume requirements, good sensitivity and selectivity, circumvents creation of enzyme-linked antibodies for every antigen of interest

Relatively fast, medium to high sample throughput, reasonably low protein and sample volume requirements

Relatively fast, medium to high sample throughput, reasonably low protein and sample volume requirements

Relatively fast, medium to high sample throughput, reasonably low protein and sample volume requirements

Relatively fast, medium to high sample throughput, reasonably low protein and sample volume requirements

Cytometric bead-based assay - multiplex capable, relatively fast, high sample

throughput

Relatively fast, medium sample throughput

Relatively fast, medium sample

throughput

Relatively fast, medium sample

throughput

Cheap, accurate, visual

Automated, relatively fast, multiple tests, medium sample throughput; low C.V.
Potential for cross-reactivity with secondary antibody, longer incubation and processing step required

Moderate to high equipment and reagent costs

Moderate to high equipment and reagent costs

Moderate to high equipment and reagent costs

Moderate to high equipment and reagent costs

Moderate to high equipment and reagent costs

Moderate to high equipment and reagent costs

Moderate to high equipment and reagent costs

Moderate to high equipment and reagent costs

Labour intensive, not readily amenable

to high-throughput applications

Moderate equipment cost

(continued on next page) 


\section{Table 1 - Continued}

\begin{tabular}{|c|c|c|c|c|}
\hline Method & References & $\begin{array}{l}\text { Immune biomarkers/clinical } \\
\text { variables measured }\end{array}$ & Advantages & Disadvantages \\
\hline & Watzl et al. (1999) ${ }^{*}$ & Total leucocyte numbers & $\begin{array}{l}\text { Automated, relatively fast, multiple tests, } \\
\text { medium sample throughput; low C.V. }\end{array}$ & Moderate equipment cost \\
\hline Nephelometry & Tang et al. (2009) & $\begin{array}{l}\text { Serum total immunoglobulins (Igs) (IgG, } \\
\text { IgA, and IgM), complement components } \\
\text { (C3 and C4), and CRP }\end{array}$ & Quick, automated, multiple tests & Moderate equipment cost \\
\hline \multirow[t]{3}{*}{$\begin{array}{l}\text { Conventional/quantitative } \\
\text { Real-Time PCR }\end{array}$} & Calabrese et al. (2000) & HIV-1 RNA & $\begin{array}{l}\text { Fast, sensitive, high sample throughput, } \\
\text { low limit of detection, can be multiplexed }\end{array}$ & $\begin{array}{l}\text { May require multiple sample } \\
\text { preparation steps, moderate equipment } \\
\text { and reagent costs }\end{array}$ \\
\hline & Savolainen et al. (2006) & $\begin{array}{l}\text { Quantitation of IL-4, IL-5, IL-10, IFN- } \\
\text { gamma and TGF-beta }\end{array}$ & $\begin{array}{l}\text { Fast, sensitive, high sample throughput, } \\
\text { low limit of detection, can be multiplexed }\end{array}$ & $\begin{array}{l}\text { May require multiple sample } \\
\text { preparation steps, moderate equipment } \\
\text { and reagent costs }\end{array}$ \\
\hline & Savolainen et al. (2007) & $\begin{array}{l}\text { Quantitation of IL-18, signalling } \\
\text { lymphocytic activation molecule, GATA- } \\
\text { 3, IL-10, and TGF-beta }\end{array}$ & $\begin{array}{l}\text { Fast, sensitive, high sample throughput, } \\
\text { low limit of detection, can be multiplexed }\end{array}$ & $\begin{array}{l}\text { May require multiple sample } \\
\text { preparation steps, moderate equipment } \\
\text { and reagent costs }\end{array}$ \\
\hline Gamma radiation measurement & See et al. (2002) & $\begin{array}{l}\text { Natural killer cell activity against 51- } \\
\text { chromium labelled MOLT-4 acute } \\
\text { lymphoblastic leukaemia cells }\end{array}$ & Sensitive & Use of radiolabels \\
\hline \multirow[t]{3}{*}{ Diary cards } & Rowe et al. (2007) & $\begin{array}{l}\text { Cold and flu symptoms (runny nose, } \\
\text { congested or stuffy nose, headache, } \\
\text { cough, sore throat, fever, nausea/ } \\
\text { vomiting, and diarrhoea) }\end{array}$ & - & Subjective \\
\hline & $\begin{array}{l}\text { Alvarez-Cuesta et al. } \\
\text { (2005) }\end{array}$ & $\begin{array}{l}\text { Pollen count, symptom and medication } \\
\text { scores }\end{array}$ & - & Subjective \\
\hline & Bergendiova et al. (2011) & $\begin{array}{l}\text { Nasal congestion, runny nose, sore } \\
\text { throat, sneezing, and cough }\end{array}$ & - & Subjective \\
\hline \multirow[t]{2}{*}{ Planimetry } & $\begin{array}{l}\text { Savolainen et al. (2006) } \\
\text { and Savolainen et al. } \\
(2007)\end{array}$ & Skin wheal following skin prick test & Fast, objective, visual & - \\
\hline & $\begin{array}{l}\text { Alvarez-Cuesta et al. } \\
\text { (2005) }\end{array}$ & Skin wheal following skin prick test & Fast, objective, visual & - \\
\hline
\end{tabular}


immune function, by examining data contained in peer-reviewed publications of human clinical trials using the most widely researched botanical ingredients for this indication. A total of 18 trials were reviewed, and in order to provide a comprehensive overview of the state-of the-art, those without placebo controls or randomisation were also considered with restrictions. Trials of this nature accounted for more than a third of those identified, thereby representing a notable proportion of studies performed in this area. The value of uncontrolled trials is however significantly diminished by a number of inherent limitations and biases. These include selection bias and the risk of knowledge of which intervention was received, which may inevitably impact on study outcome and outcome measurements. This may be particularly the case when assessing subjective outcomes, such as those based on methods for self-reporting measures. The seven uncontrolled or non-randomised trials included in this review may thus only be considered exploratory and hypothesisgenerating, with their usefulness limited to gaining insights into the potential effects or benefits of plant food supplementation in specific populations and the ability of certain experimental methods to assess these outcomes. Less confidence must therefore be placed on the findings of these studies, compared to those based on randomised, blinded, controlled trials that represent a more robust study design. While the current review was limited to the most commonly studied PFS, the number of studies identified allowed us to obtain a non-biased sample of the methods most commonly used in the evaluation of PFS benefits on the immune system. It should be noted that this review was non-systematic in nature and that a number of relevant methods may have been neglected by the exclusion of papers not related to the most commonly used PFS. Furthermore data from unpublished trials, such as that found in clinical trials registries, and trials conducted in languages other than English were not included.

Overall the general quality of studies reviewed varied from those of high quality to those that can be considered relatively poor; for studies of moderate to high quality sound conclusions could be drawn about the appropriateness and relative performance of the outcome assessment methods used. For those deemed to be of low design quality, or in instances where critical information such as detailed demographic data was missing, it was not possible to fully establish the relevance of the conclusions outlined in the study. Quite appropriately considering the regulatory status of PFS, the majority of studies employed healthy or nonpathological populations to examine the effect of PFS on biomarkers of immune disease function. Due to their regulatory status, PFS should not claim medical properties, and therefore their beneficial effects may be best demonstrated in healthy populations. Studies of healthy populations within the sample showed a higher level of quality with over $70 \%$ of reports presenting a quality score of 2 or higher, while almost $60 \%$ of studies of diseased populations had a score of 1 or less. Considering that the ratings scale used in this assessment reflects the quality of study design as well as the completeness of the reporting, this observation may indicate that the study of healthy populations is inherently better suited to PFS. Besides being out of the regulatory scope of PFS definition, the study of PFS in diseased populations is complicated by issues such as co-medication, adverse event reporting and ethical considerations around the use of a non-medicinal issued for a medical condition.

Diverse study designs were employed across the trials, the majority of which were based on a double-blind randomised controlled design. Trial designs did not appear to influence the outcome of PFS treatment effects; in all but two studies significant effects of PFS on immune function were observed. In both these non-significant studies the sample size was relatively small; in one study a sample total of 12 healthy subjects was used to assess the immune-enhancing effects against baseline measurements of preparations of S. nigra; in the second study 8 clinically obese subjects and 8 healthy age and gender-matched controls were used to investigate the effects of L. esculentum supplementation. Given the relatively small sizes of these cohorts the possibility exists that the samples were underpowered to detect any significant effects that might have been present following treatment. As highlighted in the results, and summarised in Supplementary Table 2, a number of these effects could be deemed relatively small. Both studies were thus rated low in terms of sample size quality, with only two other studies having similarly sized cohorts of 10 and 13 participants; on average 28 participants were recruited across each of the 18 studies. In addition to meeting minimum requirements for sample size, the routine inclusion of healthy subjects should also be considered. The primary benefit derived from this is a better definition of the limits of normal with respect to biochemical markers of interest, which in turn can be used to more precisely delineate the physiological effects of PFS in patients.

The prevalence or nature of adverse events (or lack thereof) observed during or after PFS exposure was not reported for the majority of studies. Adverse events nevertheless represent a major concern associated with the use of PFS, where supplement-drug interactions for example may be exacerbated under certain physiological states, or may interfere with prescription medications (Cassileth et al., 2009). Such interactions are a particularly important consideration for PFS used to enhance immune function where, due to the complexity of the immune system, increasing certain constituents may be beneficial, whilst for other constituents this could have the opposite effect (Cassileth et al., 2009). Individuals with autoimmune diseases for example, or those receiving immunosuppression medications could thus be predisposed to complications and unwanted clinical effects due to PFS-induced increases in immune function, and the reporting of adverse events during trials designed to assess PFS benefits on immune function would therefore be useful for assessing a priori the risk-benefits of such treatment.

A broad range of bioanalytical methods were identified that are suited to assessing the potential benefits of PFS on immune function. In order to provide the highest possible confidence in this selection, distinction has been made between those methods employed in randomised controlled trials and those used in open trials (see Table 1). Methods included more common approaches based on immunoassay technology, flow cytometry, PCR, and microscopy, and less frequently used methods such as nephelometry. Immunoassays proved to be the most popular method with ELISA being found to be particularly widespread. Primary advantages of 
employing immunoassays included sensitivity, throughput, and the wide range of biomarkers that can typically be detected using this technology. This included qualitative and quantitative detection of circulating white blood cells, for example monocytes and lymphocytes, levels of total immunoglobulins and immunoglobulin subclasses, cytokines and cytokine receptors, and phagocytosis and natural killer-cell activity. Statistically significant changes to the production and activity of these biomarkers were readily identified throughout different study groups undergoing treatment with a range of PFS. This capability was also observed in studies employing flow cytometry as a means of assessing benefit, for which the technology proved efficient at determining absolute counts of cells associated with innate response. These included neutrophils, monocytes, macrophages and cytokines, and lymphoctyes. Flow cytometry was found to be highly sensitive and amenable to medium-throughput use under a clinical setting. Microscopy-based methods were also identified. These were used to visually quantitate immune biomarkers, for example circulating lymphocytes; however this approach is relatively labour intensive and may generally not be amenable to processing large sample numbers in an economic manner. The use of more recent technologies such as qRT-PCR was not widespread, despite the sensitivity and diverse application potential of such approaches. In the case of qRT-PCR the technology is also amenable to high-throughput use although the associated costs can be high. Generally, the use of other relatively recent technologies such microarrays and mass spectrometry was not observed in any of the studies included in this review. Whilst the cost of implementing such technologies may be considered high, their sensitivity and throughput often translates to markedly lower costs per data point, in the process enabling a more comprehensive understanding of cause-andeffect to be gained. Such technologies may therefore prove useful in future to improving our understanding and validation of PFS benefit claims. In the case of four studies insufficient detail was given regarding the methods used to assess PFS benefit - in each of these cases the methods were referenced. Interestingly, the bioanalytical methods used to assess PFS activity seemed to vary slightly depending on the nature of the population studied, with immunoassay and flow cytometry methods being more represented in the study of healthy populations, and PCR and planimetry being more represented in the study of diseased populations. While computerised planimetry is intrinsically related to the measure of allergic reactions in sensitised individuals, the greater use of PCR in the study of diseased populations could be a spurious observation due to the limited number of studies of diseased populations included in this review. In contrast to bioanalytical methods, a number of studies employed clinical rating scales as the primary outcome measure for assessing benefit. These predominantly included the use of diaries and score cards designed to record symptomology, for example the prevalence of cold symptoms, during treatment. Very few studies employed the use of both bioanalytical and clinical approaches to simultaneously assess benefit. The use of study designs that incorporate both approaches would provide stronger support for validating claims by demonstrating correlation across multiple assessment domains.

In order to obtain a broad overview of the methods used to assess the effects of PFS on immune function, this review chose to include studies that are not based on a randomised controlled design. While such studies are widely employed, they are considered exploratory and of relatively low confidence. To gain a broader overview of the field the inclusion of methods from studies beyond the top 10 PFS may have proved useful. Few studies provided details about the validation status of their methods and the authors acknowledge that the inclusion of data from poorly validated or unvalidated methods which can affect the reliability and reproducibility of end-point outcome measurements represents a limitation. This shortfall can be circumvented by limiting studies to those that employ methods that have clearly been validated in-house, thereby increasing the likelihood that significant findings can be confirmed. Lastly, although statistical power estimates were not provided for a number of studies, the sample sizes described appeared to be relatively low in some cases to sufficiently detect the benefit effects claimed from PFS use. By limiting included studies to those that possess adequate sample power the findings and conclusions drawn from such work could be made more robust.

\section{Conclusions}

At total of 9 distinct methods were identified in this review for assessing the potential benefits of PFS on human immune function. Despite wide differences in the underlying principle of the approaches used, the performance of each was found to be suited to analysing biomarkers, or clinical symptoms, associated with the effects of PFS on the immune function of healthy as well as diseased individuals. The appropriateness of each method was generally limited to assessing specific biomarkers or symptoms, and patient sample size and the overall quality of the study design appeared to influence trial outcome. The use of additional modern analytical methods, combined with the use of adequately powered cohorts and improvements in outcomes reporting would contribute greatly towards advancing existing knowledge and understanding of the benefits of PFS on human immune function.

\section{Funding}

Funding for this work was received from the European Community's Seventh Framework Programme (FP7/2007-2013) under grant agreement No. 245199, as part of the PlantLIBRA project (website: www.plantlibra.eu). The review does not necessarily reflect the Commission's views or its future policy in this area. Additional funding was provided by the Department of Science and Technology, and the CSIR, South Africa.

\section{Appendix A. Supplementary data}

Supplementary data associated with this article can be found, in the online version, at http://dx.doi.org/10.1016/j.jff.2013.07. 008. 
REFERENCES

Alvarez-Cuesta, E., Aragoneses-Gilsanz, E., Martin-Garcia, C., Berges-Gimeno, P., Gonzalez-Mancebo, E., \& Cuesta-Herranz, J. (2005). Immunotherapy with depigmented glutaraldehydepolymerized extracts: Changes in quality of life. Clinical and Experimental Allergy: Journal of the British Society for Allergy and Clinical Immunology, 35, 572-578.

Barak, V., Birkenfeld, S., Halperin, T., \& Kalickman, I. (2002). The effect of herbal remedies on the production of human inflammatory and anti-inflammatory cytokines. The Israel Medical Association Journal: IMAJ, 4, 919-922.

Beltran-Debon, R., Alonso-Villaverde, C., Aragones, G., RodriguezMedina, I., Rull, A., Micol, V., et al. (2010). The aqueous extract of Hibiscus sabdariffa calices modulates the production of monocyte chemoattractant protein-1 in humans. Phytomedicine: International Journal of Phytotherapy and Phytopharmacology, 17, 186-191.

Bergendiova, K., Tibenska, E., \& Majtan, J. (2011). Pleuran (betaglucan from Pleurotus ostreatus) supplementation, cellular immune response and respiratory tract infections in athletes. European Journal of Applied Physiology, 111, 2033-2040.

Bobovcak, M., Kuniakova, R., Gabriz, J., \& Majtan, J. (2010). Effect of pleuran (beta-glucan from Pleurotus ostreatus) supplementation on cellular immune response after intensive exercise in elite athletes. Applied Physiology, Nutrition, and Metabolism $=$ Physiologie Appliquee, Nutrition Et Metabolisme, 35, 755-762.

Bonilla, F. A., \& Oettgen, H. C. (2010). Adaptive immunity. The Journal of Allergy and Clinical Immunology, 125, S33-S40.

Briviba, K., Kulling, S. E., Moseneder, J., Watzl, B., Rechkemmer, G., \& Bub, A. (2004). Effects of supplementing a lowcarotenoid diet with a tomato extract for 2 weeks on endogenous levels of DNA single strand breaks and immune functions in healthy non-smokers and smokers. Carcinogenesis, 25, 2373-2378.

Calabrese, C., Berman, S. H., Babish, J. G., Ma, X., Shinto, L., Dorr, M., et al. (2000). A phase I trial of andrographolide in HIV positive patients and normal volunteers. Phytotherapy Research: PTR, 14, 333-338.

Canter, P. H., \& Ernst, E. (2004). Herbal supplement use by persons aged over 50 years in britain: Frequently used herbs, concomitant use of herbs, nutritional supplements and prescription drugs, rate of informing doctors and potential for negative interactions. Drugs \& Aging, 21, 597-605.

Cassileth, B. R., Heitzer, M., \& Wesa, K. (2009). The public health impact of herbs and nutritional supplements. Pharmaceutical Biology, 47, 761-767.

DeGrella, R. F., Combs, G. L., Coffee, E. E., Stern, C. H., Frenkel, D., Kureshy, F., et al. (1985). A nephelometry system for the abbott TDx analyzer. Clinical Chemistry, 31, 1474-1477.

Dessein, R., Chamaillard, M., \& Danese, S. (2008). Innate immunity in crohn's disease: The reverse side of the medal. Journal of Clinical Gastroenterology, 42(Suppl 3 Pt 1), S144-S147.

Di Meglio, P., Perera, G. K., \& Nestle, F. O. (2011). The multitasking organ: Recent insights into skin immune function. Immunity, 35, 857-869.

Engvall, E., \& Perlmann, P. (1971). Enzyme-linked immunosorbent assay (ELISA). Quantitative assay of immunoglobulin G. Immunochemistry, 8, 871-874.

European Commission. (2002). EC (2002) directive 2002/46/EC of the european parliament and of the council of 10 june 2002 on the approximation of the laws of the member states relating to food supplements. European Parliament.

Finn, P. W., \& Bigby, T. D. (2009). Innate immunity and asthma. Proceedings of the American Thoracic Society, 6, 260-265.
Gleeson, M. (2005). Assessing immune function changes in exercise and diet intervention studies. Current Opinion in Clinical Nutrition and Metabolic Care, 8, 511-515.

Hughes, D. A. (1999). Effects of carotenoids on human immune function. The Proceedings of the Nutrition Society, 58, 713-718.

Ibs, K. H., \& Rink, L. (2003). Zinc-altered immune function. The Journal of Nutrition, 133, 1452S-1456S.

Karlsen, A., Paur, I., Bohn, S. K., Sakhi, A. K., Borge, G. I., Serafini, M., et al. (2010). Bilberry juice modulates plasma concentration of NF-kappaB related inflammatory markers in subjects at increased risk of CVD. European Journal of Nutrition, 49, 345-355.

Karlsen, A., Retterstol, L., Laake, P., Paur, I., Kjolsrud-Bohn, S., Sandvik, L., et al. (2007). Anthocyanins inhibit nuclear factorkappaB activation in monocytes and reduce plasma concentrations of pro-inflammatory mediators in healthy adults. The Journal of Nutrition, 137, 1951-1954.

Kiely, M., Black, L. J., Plumb, J., Kroon, P. A., Hollman, P. C., Larsen, J. C., et al. (2010). EuroFIR eBASIS: Application for health claims submissions and evaluations. European Journal of Clinical Nutrition, 64(Suppl. 3), S101-S107.

Kim, H. Y., DeKruyff, R. H., \& Umetsu, D. T. (2010). The many paths to asthma: Phenotype shaped by innate and adaptive immunity. Nature Immunology, 11, 577-584.

Lomax, A. R., \& Calder, P. C. (2009). Prebiotics, immune function, infection and inflammation: A review of the evidence. The British Journal of Nutrition, 101, 633-658.

Markovits, N., Ben Amotz, A., \& Levy, Y. (2009). The effect of tomato-derived lycopene on low carotenoids and enhanced systemic inflammation and oxidation in severe obesity. The Israel Medical Association Journal: IMAJ, 11, 598-601.

Mizuno, M. (2006). A review of current knowledge of the complement system and the therapeutic opportunities in inflammatory arthritis. Current Medicinal Chemistry, 13, 1707-1717.

Mullis, K., Faloona, F., Scharf, S., Saiki, R., Horn, G., \& Erlich, H. (1986). Specific enzymatic amplification of DNA in vitro: The polymerase chain reaction. Cold Spring Harbor Symposia on Quantitative Biology, 51(Pt. 1), 263-273.

Myers, S. P., Stevenson, L., Cheras, P. A., O'Connor, J., Brooks, L., Rolfe, M., et al. (2010). A forced titration study of the antioxidant and immunomodulatory effects of ambrotose AO supplement. BMC Complementary and Alternative Medicine, 10, 16.

Obermayer-Straub, P., Strassburg, C. P., \& Manns, M. P. (2000). Autoimmune polyglandular syndrome type 1. Clinical Reviews in Allergy \& Immunology, 18, 167-183.

O'Leary, A. (1990). Stress, emotion, and human immune function. Psychological Bulletin, 108, 363-382.

Parham, P. (2009). The immune system (3rd ed.). United Kingdom: Garland Science.

Pearson, T., Greiner, D. L., \& Shultz, L. D. (2008). Humanized SCID mouse models for biomedical research. Current Topics in Microbiology and Immunology, 324, 25-51.

Plat, J., \& Mensink, R. P. (2005). Food components and immune function. Current Opinion in Lipidology, 16, 31-37.

Pothoulaki, M., \& Chryssochoidis, G. (2009). Health claims: Consumers' matters. Journal of Functional Foods, 1, 222-228.

Riso, P., Visioli, F., Grande, S., Guarnieri, S., Gardana, C., Simonetti, P., et al. (2006). Effect of a tomato-based drink on markers of inflammation, immunomodulation, and oxidative stress. Journal of Agricultural and Food Chemistry, 54, 2563-2566.

Rowe, C. A., Nantz, M. P., Bukowski, J. F., \& Percival, S. S. (2007). Specific formulation of Camellia sinensis prevents cold and flu symptoms and enhances gamma, delta T cell function: A randomized, double-blind, placebo-controlled study. Journal of the American College of Nutrition, 26, 445-452. 
Savolainen, J., Jacobsen, L., \& Valovirta, E. (2006). Sublingual immunotherapy in children modulates allergen-induced in vitro expression of cytokine mRNA in PBMC. Allergy, 61, 1184-1190.

Savolainen, J., Nieminen, K., Laaksonen, K., Laiho, T., Jacobsen, L., Lahesmaa, R., et al. (2007). Allergen-induced in vitro expression of IL-18, SLAM and GATA-3 mRNA in PBMC during sublingual immunotherapy. Allergy, 62, 949-953.

See, D., Mason, S., \& Roshan, R. (2002). Increased tumor necrosis factor alpha (TNF-alpha) and natural killer cell (NK) function using an integrative approach in late stage cancers. Immunological Investigations, 31, 137-153.

Sly, P. D., \& Holt, P. G. (2011). Role of innate immunity in the development of allergy and asthma. Current Opinion in Allergy and Clinical Immunology, 11, 127-131.

Tang, Y. P., Li, P. G., Kondo, M., Ji, H. P., Kou, Y., \& Ou, B. (2009). Effect of a mangosteen dietary supplement on human immune function: A randomized, double-blind, placebocontrolled trial. Journal of Medicinal Food, 12, 755-763.

Turvey, S. E., \& Broide, D. H. (2010). Innate immunity. The Journal of Allergy and Clinical Immunology, 125, S24-S32.

Van Heel, D. A., McGovern, D. P., \& Jewell, D. P. (2001). Crohn's disease: Genetic susceptibility, bacteria, and innate immunity. Lancet, 357, 1902-1904.

Watzl, B., Bub, A., Brandstetter, B. R., \& Rechkemmer, G. (1999). Modulation of human T-lymphocyte functions by the consumption of carotenoid-rich vegetables. The British Journal of Nutrition, 82, 383-389.

Webster Marketon, J. I., \& Glaser, R. (2008). Stress hormones and immune function. Cellular Immunology, 252, 16-26.

Wood, L. G., Garg, M. L., Powell, H., \& Gibson, P. G. (2008). Lycopene-rich treatments modify noneosinophilic airway inflammation in asthma: Proof of concept. Free Radical Research, 42, 94-102. 